科 学 通 报

\title{
一类拟线性方程组高维黎曼问题及其扰动 *
}

\author{
陈恕行 \\ (复旦大学数学系, 上海 200433)
}

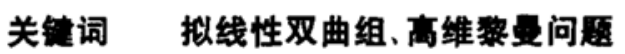

在本文中我们讨论拟线性方程组

$$
U_{t}+F(U)_{x}+G(U)_{y}=0
$$

的柯西问题. 其中 $U=^{t}(u, v), F={ }^{t}\left(u^{2}, u v\right), G={ }^{t}\left(u v, v^{2}\right)$, 这个方程组是非严格双曲的. 它可能 在石油开采、弹性理论等物理问题中遇到, 问题的初始条件给定为

$$
\left.U\right|_{t=0}=U^{0}(x, y) \text {, }
$$

其中 $U^{0}(x, y)$ 在过原点的三条曲线 $\Gamma_{i}$ 上有间断, 在由 $\Gamma_{i}$ 所分成的区域 $\Omega_{\mathrm{i}}$ 上分别为光滑函数组 $U_{i}$ $(x, y)$. 这个问题的解一般将具有一个发自原点的花状奇性结构, 它不能视为一维问题的扰 动, 从而称为本性高维问题. 当初始条件为分块常数且 $\Gamma_{\mathrm{i}}$ 为直线时, 问题又称为高维黎曼问 题. 高维黎曼问题首先在文献 [1] 中被提出; 在文献 [2-4 $]^{11}$ 中已对该问题的自模解作了初步的研究.

本文将在较一般的假定条件下讨论问题 (1),(2) 式的解. 我们仅要求初始条件中 $v^{0} / u^{0}$ 为 分块常数,因而所得到的解一般来说并非自模解, 然而它可以作为自模解的扰动来得到.

\section{1 一维问题的扰动}

若初始资料 $\left.U\right|_{t=0}$ 仅在过原点的一条直线上有间断, 则问题是一维的. 因为方程组 (1) 在 坐标系的旋转变换下不变, 故我们可设初始资料具有形式为

$$
\left.U\right|_{t=0}= \begin{cases}\left(u^{+}, v^{+}\right), & x>0, \\ \left(u^{-}, v^{-}\right), & x<0 .\end{cases}
$$

若 $u^{+}>u^{-}>0$, 则得到接触间断与单波的结构 $\left(J-R\right.$ 结构); 又若 $u^{-}>u^{+}>0$, 则得到接触间断 与激波的结构 $\left(J-S\right.$ 结构), 但是在 $u^{+} u^{-}<0$ 时仅含初等波的解可能不存在.

若在初始平面上, $U^{0}$ 在曲线 $\Gamma: x=g(y)$ 上有间断, 则解不是自模的. 注意到从(1) 式可以 导出方程 $\left(\partial_{t}+u \partial_{x}+v \partial_{y}\right)(v / u)=0$; 故若在 $\Omega$ 中 $v^{0} / u^{0}=c$, 则在 $\Omega$ 在决定区域中也有 $v / u=c$, 并利 用特征线法可知在此决定区域中局部解存在.

对于初始资料的间断线的邻域, 若冻结问题的解是 $J-S$ 结构, 将在 $J$ 的左、右边的解分别 记为 $U_{a}$ 与 $U_{b}$, 则 $U_{a}$ 已知, 且 $J: x=j(t, y)$ 的方程由

$$
j_{t}-u_{a}+j_{y} v_{a}=0, j(0, y)=g(y)
$$

1993-08-08 收稿.

*国家自然科学基金, 天元基金与国家教委博士点基金资助项目.

1) Tan, D. C., Zhang, T., Two Dimensional Riemann Problem for a Hyperbolio System of Nonlinear Conservation Lows, (1): Four J Cases, Preprint. 
决定, 接着可决定 $U_{b}$, 并由

$$
s_{t}-\left(u_{b}+u_{c}\right)+s_{y}\left(v_{b}+v_{c}\right)=0, s(0, y)=g(y)
$$

决定激波 $S: x=s(t, y)$. 其中 $u_{c}$ 是由 $\Gamma$ 左侧的初值决定的解. 总之, 含 $J-S$ 结构的解为

$$
U(t, x, y)=\left\{\begin{array}{l}
U_{a}(t, x, y), \text { 若 } x<j(t, y), \\
U_{b}(t, x, y), \text { 若 } j(t, y)<x<s(t, y), \\
U_{c}(t, x, y), \text { 若 } s(t, y)<x .
\end{array}\right.
$$

若冻结问题的解具 $J-R$ 结构, 则解为

$$
U(t, x, y)=\left\{\begin{array}{l}
U_{a}(t, x, y), \text { 若 } x<j(t, y), \\
U_{b}(t, x, y), \text { 若 } j(t, y)<x<\pi_{1}(t, y), \\
U_{r}(t, x, y), \text { 若 } \pi_{1}(t, y)<x<\pi_{0}(t, y), \\
U_{c}(t, x, y), \text { 若 } \pi_{0}(t, y)<x,
\end{array}\right.
$$

其中 $x=\pi_{0}(t, y), x=\pi_{1}(t, y)$ 是过 $x=g(y)$ 的第二族特征面, 它代表单波 $R$ 的前阵面与后阵 面, 夹在这两个曲面之间的单波解 $U_{r}$ 是利用给定在 $\Gamma$ 上的含双参数的初值 $\widetilde{U}(\theta, y)=\theta$ $U_{b}+(1-\theta) U_{2}$ 来决定的, 且每个 $\theta \in[0,1]$ 对应于一个第二族特征曲面.

上面所述的具 $J-R$ 或 $J-S$ 结构的解关于初值的扰动是稳定的, 只要这个扰动仍使 $v^{0} / u^{0}$ 为 分块常数.

\section{2 高维问题自模解的局部结构}

如果将初始条件 (2) 在原点处作平坦化处理, 即把曲线 $\Gamma_{i}$ 更换为过原点的切线 $l_{i}$ : $\theta=\theta_{i}$, 把 $U_{i}(x, y)$ 更换为它们在原点的极限值 $U_{i o}$. 则可得到方程组 (1) 的一个取冻结初始资 料 $\left.U\right|_{t=0}=U_{i o}\left(\theta_{i-1}<\theta<\theta_{i}\right)$ 的柯西问题. 这个问题的解是自模的. 即有 $U(t, x, y)=U(\xi, \eta)$, 其 中 $\xi=x / t, \eta=y / t$. 在 $(\xi, \eta)$ 平面上, 除方程组需作变形外, 初始条件也化成当 $r \rightarrow \infty$ 时在方 向 $\theta_{i}$ 上 $U$ 的值为已知, 为作出冻结问题在 $(\xi, \eta)$ 平面上的解, 需要先分析初等波干扰的局部 结构. 为此, 我们引人结点的概念.

定义 若在 $(\xi, \eta)$ 平面上, 解 $U$ 的多条间断线交于 $Q$, 考察在 $Q$ 点邻域中 $U$ 的平坦化. 我们称 $Q$ 为合理的非平凡结点, 若以下条件满足:

(i) $U=W_{j}\left(\alpha_{j-1}<\alpha<\alpha_{j}\right)$ 其中 $\alpha$ 为出发自 $Q$ 点的射线与水平线之夹角, $W_{j}$ 是常数或是中心波.

(ii) 在 $U$ 的间断线上满足 $R-H$ 条件, 且在激波线上满足嫡条件.

(iii) 在 $Q$ 的邻域中 $U \neq(0,0)$.

(iv) 在 $Q$ 的邻域中至多有两个波是进人的. 且进人波必是相邻的.

定理 1 满足以上假定条件的结点必定属于下面四种类型之一:

1) $S S S$, 其中两个 $S$ 为到来, 一个为离去.

2) $J S J S$, 其中 $S$ 一个为到来, 一个为离去.

3) $J J S$ 或 $J J S J$, 其中 $S$ 为离去.

4) $J J R$, 其中 $R$ 为离去.

基于上述对结点的分类, 可以完整地给出初等波干扰的局部结构. 按参与干扰的波加以 分类, 共有以下五种情形:

(a) $R \otimes S$ : 在 $(\xi, \eta)$ 平面上 $R$ 与 $S$ 相遇并不产生非平凡结点. 两波相遇后, 激波割去单 
波, 并改变其方向继续传播. 记激波与单波的前阵面之交点为 $\left(\xi_{Q}, \eta_{Q}\right)$, 则相交后的激波可由

$$
\frac{\mathrm{d} \eta}{\mathrm{d} \xi}=\frac{\eta-v_{+}-v_{R}}{\xi-u_{+}-u_{R}},\left.\eta\right|_{\xi=\xi_{Q}}=\eta_{Q}
$$

决定, 其中 $\left(u_{R}, v_{R}\right)$ 为 单波区域的解, $\left(u_{+}, v_{+}\right)$为激波外侧的解.

(b) $R \otimes J$ : 这时也不出现非平凡结点. 当两波相遇后, $R$ 与 $J$ 均将越过对方而继续传播, 且分别改变其方向. $J$ 波穿人单波区域时的方程由下式决定：

$$
\frac{\mathrm{d} \eta}{\mathrm{d} \xi}=\frac{\eta-v}{\xi-u},\left.\eta\right|_{\xi=\xi_{Q}}=\eta_{Q} \text {. }
$$

(c) $S \otimes S$ : 两个激波的交点为非平凡结点, 它相应于定理 1 中的情形 1). 相交后两个激波 合成一个新的激波, 其方程由 (8) 式描述.

(d) $S \otimes J$ : 激波与接触间断之交点为非平凡结点, 它相应于定理 1 中的情形 2). 这时 $S$ 波 与 $J$ 波越过对方继续传播, 它们的方程分别由 (8) 与 (9)式决定.

(e) $J \otimes J$ : 两个接触间断之交点也是非平凡结点. 如果在两个 $J$ 波的外侧状态位于过原 点的同一射线上, 则根据这些状态在相平面中的位置, 结点对应于 $J J S$ 或 $J J R$ 的情形. 又如果 在两个 $J$ 波的外侧状态不在过原点的同一射线上, 则或者该结点属于 $J J S J$ 的结构, 或者这种 局部解结构不可能出现.

\section{3 高维问题自模解的整体构造及其扰动}

以解的局部结构的分析为基础, 可以构造整体自模解. 由于初值在 $\theta=\theta_{i}(i=1,2,3)$ 上 有间断, 故在 $\theta=\theta_{i}$ 方向上有到来波. 我们允许每个方向有两个到来波,故按从无穷远处到来 的波来区分, 解的整体结构共有 $J S-J S-J S, J S-J S-J R, J S-J R-J R, J R-J R-J R$ 四大类. 这种解均 称为具有完全奇性结构的解. 在构造整体自 模解时可以先作出所有的 $J$ 波, 因为 $J$ 波个 数在干扰中只能减少而不能增加. 接着再作 $R$ 波与 $S$ 波. 图 1中给出了 $J S-J S-J R$ 情形 下解的奇性结构的一个实例.

最后我们考察原始问题 (1), (2) 式. 一 般来说, 它的解是非自模的. 但由于方程 (1) 的解 $U(t, x, y)$ 经过伸缩变换 $t \rightarrow \alpha t, x$ $\rightarrow \alpha x, y \rightarrow \alpha y$ 以后仍为解, 而其在初始平面上 的间断线 $\widetilde{\Gamma}_{i}: \theta=\theta_{i}(r)$ 将变换成 $\Gamma_{i}: \theta=\theta_{i}(\alpha r)$, 故 取 $\alpha$ 充分小可以使在确定的有限区域中 $\widetilde{\Gamma}_{i}$ 几近于直线, 且 $U_{i}$ 也几近于常数. 故若限 于在原点充分小的邻域中考察局部解, 原问 题可视为冻结问题的微扰. 由于一维问题 的解以及上节中所讨论的波的局部干扰对 于扰动都是稳定的, 从而原问题的非自模解 可以通过由冻结问题的自模解出发, 经过一

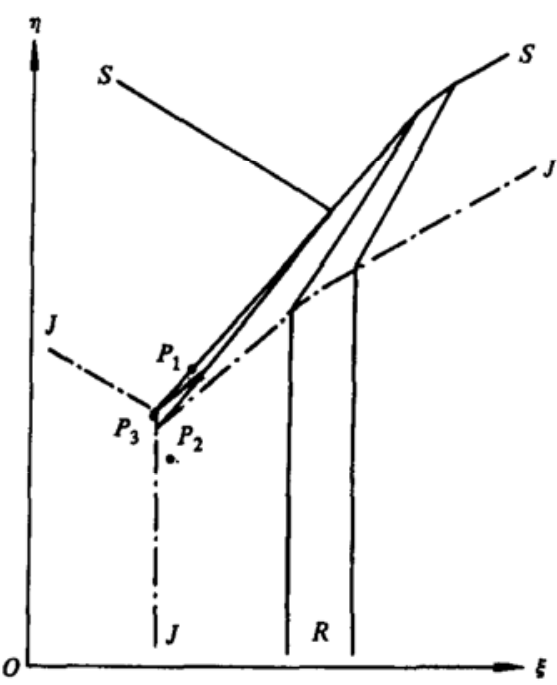

图 1 其中 $P_{i}$ 表示 $U_{i}$ 在相平面上的位置 
系列稳定的扰动得到. 由此可得

定理 2 若问题 (1), (2) 式相应的冻结问题有具有完全结构的自模解, 则在原点的邻域中 该原始问题的局部解也存在,而且它是冻结问题解的扰动.

\section{参考文献}

[1] Courant, R., Friedrichs, K. O., Supersonic Flow and Shock Waves, Interscience Publishers Inc.; New York, 1948.

[2] Wagner, D., Math. Ann., 1983, 14:534-559.

[ 3] Lindquist, B., SIAM J. Anal., 1986, 14:1178- 1197.

[4] Zhang, T., Xiao, L., Pitman Monograph \& Surveys, 1989, 41. 